\title{
ESTABILIDAD OXIDATIVA DE LOS ACEITES DE SACHA INCHI (PLUKENETIA HUAYLLABAMBANA Y PLUKENETIA VOLUBILIS) Y SU IMPORTANCIA EN LA INDUSTRIA ALIMENTARIA
}

NANCY CHASQUIBOL* https://orcid.org/0000-0002-9275-2060 Centro de Estudios e Innovación de Alimentos Funcionales de la Facultad de Ingeniería y Arquitectura, Universidad de Lima, Perú Instituto de Investigación Científica (IDIC), Universidad de Lima, Perú

KAREN IPARRAGUIRRE https://orcid.org/0000-0002-0547-4606 Centro de Estudios e Innovación de Alimentos Funcionales de la Facultad de Ingeniería y Arquitectura, Universidad de Lima, Perú

JOSÉ HUAMÁN

https://orcid.org/0000-0002-3725-0455 Centro de Estudios e Innovación de Alimentos Funcionales de la Facultad de Ingeniería y Arquitectura, Universidad de Lima, Perú

RAfael Alarcón https://orcid.org/0000-0003-3315-9467 Centro de Estudios e Innovación de Alimentos Funcionales de la Facultad de Ingeniería y Arquitectura, Universidad de Lima, Perú

Recibido: 12 de mayo del 2019 / Aprobado: 9 de junio del 2020

doi: https://doi.org/10.26439/ing.ind2020.n039.4922

RESUMEN: El objetivo de este trabajo es determinar la estabilidad oxidativa de los aceites de sacha inchi (Plukenetia huayllabambana y Plukenetia volubilis) sin microencapsular y microencapsulados con diferentes materiales de pared, empleando el método de Rancimat a $70^{\circ} \mathrm{C}, 80^{\circ} \mathrm{C}, 90^{\circ} \mathrm{C}$ y $100^{\circ} \mathrm{C}$ y la técnica de extrapolación lineal en función de los periodos de inducción se estimaron los tiempos de vida útil a $25^{\circ} \mathrm{C}$. Se realizaron los siguientes análisis físico-químicos: índice de acidez, índice de peróxidos, estabilidad oxidativa y humedad.

Palabras clave: aceite de sacha inchi / estabilidad oxidativa / método Rancimat / microcápsulas / vida útil de aceites de sacha inchi

*Correo electrónico: nchasquibol@ulima.edu.pe 


\title{
OXIDATIVE STABILITY OF SACHA INCHI OILS \\ (PLUKENETIA HUAYLLABAMBANA AND \\ PLUKENETIA VOLUBILIS) AND THEIR IMPORTANCE IN THE FOOD INDUSTRY
}

\begin{abstract}
This work aims to determine the oxidative stability of sacha inchi oils (Plukenetia huayllabambana and Plukenetia volubilis), both non-microencapsulated and microencapsulated with different wall materials, using the Rancimat method at 70 , 80,90 and $100^{\circ} \mathrm{C}$, and the linear extrapolation technique based on induction periods. Shelf-life values were estimated at $25^{\circ} \mathrm{C}$. The following physical and chemical analysis were carried out: acidity level, peroxide index, oxidative stability and humidity.
\end{abstract}

Keywords: sacha inchi oil / oxidative stability / Rancimat method / microcapsules / shelf life of sacha inchi oils 


\section{INTRODUCCIÓN}

Los lípidos, proteínas y carbohidratos, entre otros, son nutrientes importantes por sus beneficios nutricionales y funcionales. Los lípidos (representados mayoritariamente por aceites y grasas) juegan un papel importante en la dieta humana por su aporte en ácidos grasos esenciales y vitaminas liposolubles.

Los aceites vegetales están compuestos por ácidos grasos saturados e insaturados, como los ácidos grasos poliinsaturados o ácidos grasos esenciales ( $\omega-3, \omega-6$ y $\omega-9$ ). El cuerpo humano no los biosintetiza; por ello, es necesario obtenerlos mediante los alimentos o en forma de suplementos. Las fuentes más importantes de $\omega$-3 se encuentran en productos hidrobiológicos, semillas vegetales, entre otros (Ancira, 2008).

El aceite de sacha inchi, de la especie Plukenetia volubilis, es muy valorado por sus cualidades funcionales, debido a su alto contenido de ácidos grasos $\omega-3[42,00-48,00 \%$ a-ácido linolénico (Ln)] (Norma Técnica Peruana NTP 151.400, 2014). Además, posee una composición particular de esteroles (estigmasterol>campesterol) y un alto porcentaje de $\gamma$ - y $\delta$-tocoferoles $\left(>1,900 \mathrm{mg} \cdot \mathrm{kg}^{-1}\right)$; en comparación con otras semillas oleaginosas (Guillén et al., 2003; Bondioli et al., 2006; Chasquibol et al., 2012; Liua et al., 2014). Es un aceite que se puede consumir crudo o virgen, sin otro procesado más que su extracción mediante prensado en frío. Además, es muy apreciado por sus propiedades beneficiosas para la salud (Gorriti et al., 2010; Huamán et al., 2008; Garmendia et al., 2011; Gonzales et al., 2014) por sus singulares características de olor y sabor. El ecotipo Plukenetia huayllabambana es otra especie que presenta granos de mayor tamaño y con mayor contenido de $\omega$-3 (55,62 a 60,42 \% a-ácido linolénico - Ln) (Chasquibol et al., 2014) que la especie comercial (Plukenetia volubilis). Crece en estado silvestre en la provincia de Rodríguez de Mendoza, departamento de Amazonas, Perú y existe escasa información científica sobre esta especie (Muñoz et al., 2013).

Los aceites de sacha inchi, por su naturaleza química, presentan una alta susceptibilidad a la oxidación que limita su vida útil y ocasiona una pérdida en su valor nutricional, así como la formación de compuestos indeseables; por ello, es necesario utilizar tecnologías industriales como la microencapsulación para mejorar su vida útil y la conservación de su composición química, así como de sus propiedades funcionales.

El proceso de microencapsulación por el método de secado por aspersión (spray drying) es una tecnología de empaquetamiento que emplea diferentes materiales de pared (gomas, almidones, proteínas, entre otros) que permiten retardar las reacciones oxidativas e incrementar la vida útil de los aceites, disminuyendo significativamente los efectos del proceso de oxidación lipídica. Existen diferentes métodos para la determinación de la estabilidad oxidativa de los aceites. Uno de los más empleados es el de Rancimat (Cabré y Massó, 2014; Páucar et al., 2015; Rodríguez et al., 2015; Villanueva 
et al., 2013), el cual fue empleado en el presente trabajo para determinar el tiempo de vida útil de los aceites de sacha inchi (Plukenetia volubilis y Plukenetia huayllabambana) sin microencapsular y microencapsulados.

Según Chasquibol (2017), el proceso de oxidación de los lípidos ocurre fundamentalmente por la existencia de los ácidos grasos insaturados a través de una serie de reacciones en cadena de radicales libres. La autooxidación, es la principal reacción implicada en el deterioro de los lípidos. Existen dos tipos de variables que afecta a la oxidación de los aceites y grasas:

- Variable extrínseca. Relacionada con las condiciones de almacenamiento, como temperatura, exposición a la luz y disponibilidad de oxígeno.

- Variable inherente. Relacionada con los componentes propios del aceite como los ácidos grasos, triglicéridos y componentes menores (antioxidantes y prooxidantes).

Algunas variables extrínsecas se pueden controlar de manera parcial o total. Por ejemplo, para evitar la exposición a la luz, muchos aceites son envasados en envases opacos o coloreados y se guardan en cajas opacas; para evitar la exposición al oxígeno, se envasa en atmósfera de nitrógeno y se utilizan envases de baja permeabilidad. El objetivo es prolongar la vida útil de los aceites y grasas cuando estos están envasados y mantener la calidad de estos.

La autooxidación es la reacción del oxígeno molecular atmosférico con los ácidos grasos que constituyen los triglicéridos. En el proceso de autooxidación, se denomina período de inducción (PI) o tiempo de inducción al tiempo durante el cual hay una formación escasa o indetectable de peróxidos hasta cuando se produce un incremento brusco en la oxidación.

Algunos productos primarios de la autooxidación lipídica son los hidroperóxidos, estos son relativamente inestables e intervienen en numerosas y complejas reacciones de ruptura e interacción, que son las responsables de la producción de muchos tipos de compuestos de distinta naturaleza química y variados pesos capaces de producir aromas biológicamente significativos. La autooxidación es una reacción en cadena de radicales libres y consta de tres etapas: iniciación o inducción, propagación y terminación.

La etapa de iniciación o inducción consiste en la sustracción de un hidrógeno metilénico adyacente a un doble enlace en una molécula lipídica, en presencia de un iniciador (I) para formar un radical alquilo () como se muestra a continuación:

$$
L H+I \rightarrow L \bullet+I+H \bullet \ldots(\mathrm{I})
$$


La reacción $\mathrm{LH}+\mathrm{O}_{2}$, para formar radicales libres, es termodinámicamente impedida por su elevada energía de activación ( $35 \mathrm{Kcal} / \mathrm{mol}$ ); por ello, la aparición de los primeros radicales libres necesarios para iniciar la propagación de la reacción se produce por medio de un catalizador.

En la etapa de propagación, el radical alquilo ( $L \bullet$ producido en la etapa de iniciación, reacciona con el oxígeno para formar un radical peroxilo (LOO•). Este, a su vez, reacciona con un lípido insaturado para formar un hidroperóxido y un nuevo radical libre $(L \bullet \cdot)$. Este último puede reaccionar con el oxígeno para formar un radical peroxilo, y así sucesivamente, como se muestra a continuación:

$$
\begin{gathered}
\mathrm{L} \bullet+\mathrm{O}_{2} \stackrel{k_{o}}{\rightarrow} \mathrm{LOO} \bullet \ldots(\mathrm{II}) \\
\mathrm{LOO} \bullet+\mathrm{LH} \stackrel{k_{p}}{\rightarrow} \mathrm{LOOH}+\mathrm{L} \bullet \ldots
\end{gathered}
$$

Una vez formado el número suficiente de radicales libres (I), la reacción en cadena se propaga al captar átomos de hidrógeno de las posiciones alfa (primer átomo unido al grupo funcional) de los dobles enlaces. Posteriormente, en estas posiciones se produce la adición de oxígeno, dando lugar a la producción de radicales peroxilo $L O O$ • (II), que a su vez captan hidrógeno de los grupos a-metilénicos de otras moléculas para dar hidroperóxidos $(\mathrm{LOOH})$ y grupos $(\mathrm{L} \bullet)(\mathrm{III})$. Estos, a su vez, reaccionan con el oxígeno, repitiéndose la secuencia de reacción.

Finalmente, en la etapa de terminación finaliza el proceso de oxidación, ya sea esta negativa o positiva. Si es negativa, los antioxidantes se han agotado y el proceso de oxidación continuará. Si es positiva, los antioxidantes (actúa como un atrapante de radicales libres) posiblemente han detenido el progreso de la oxidación. La velocidad de la reacción en cadena disminuye tras alcanzar un máximo. Los radicales reaccionan entre sí eliminándose y dando lugar a la formación de productos no radicalarios de estabilidad muy variada, entre los que se encuentran:

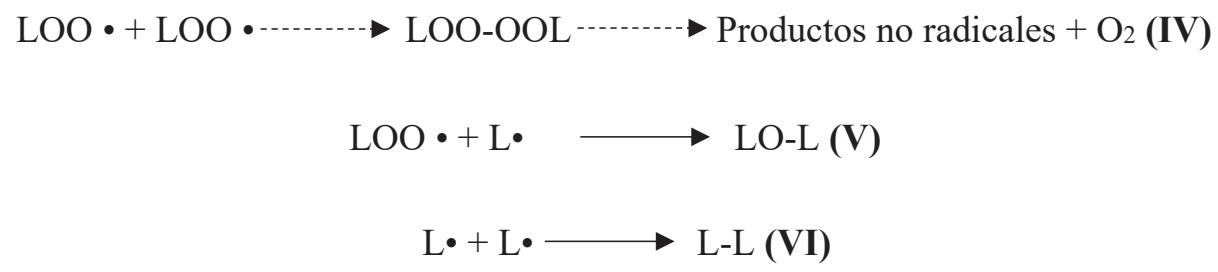

Como consecuencia de las reacciones de terminación, aparecen productos oxidados estables y algunos volátiles originados por rotura de las cadenas acídicas. En el período de inducción, el consumo de oxígeno es lento y aumenta rápidamente cuando tiene lugar 
la formación de los compuestos de terminación. El proceso de oxidación se muestra en la figura 1.

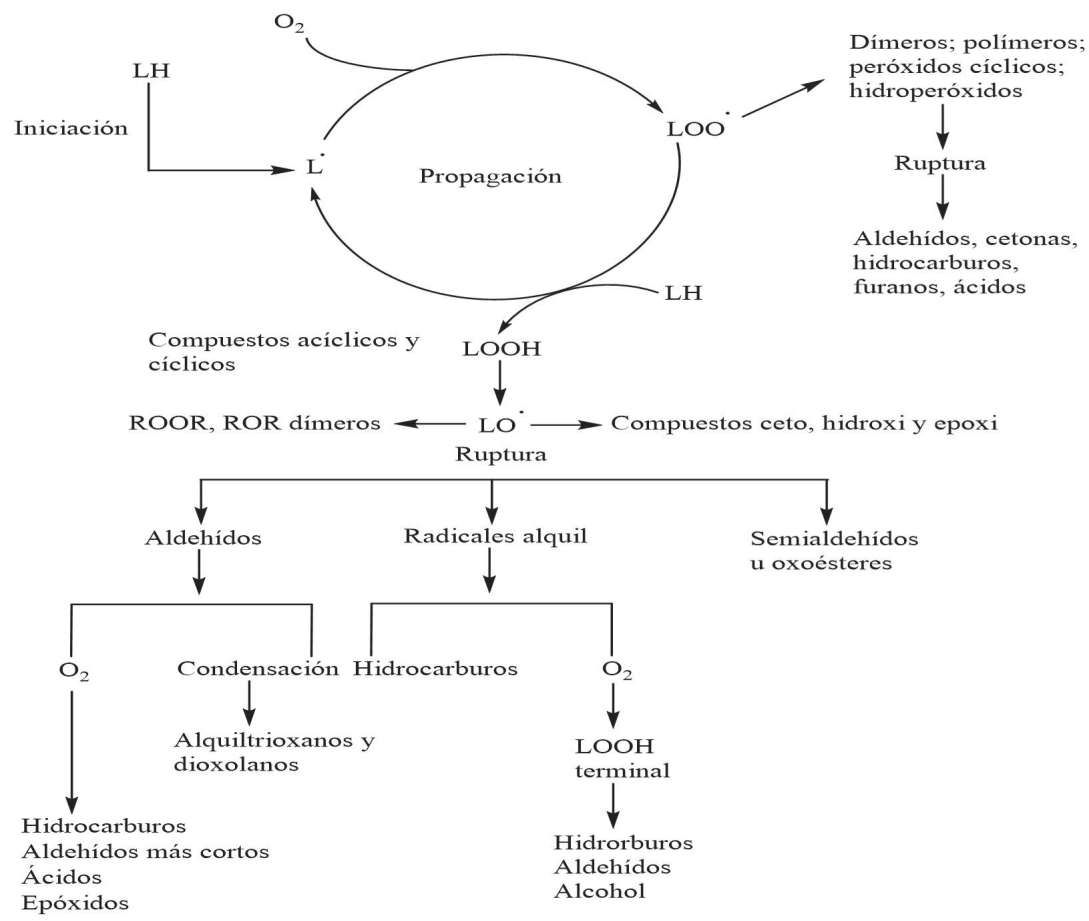

Figura 1. Mecanismo de autoxidación y los productos que se obtienen a partir de los hidroperóxidos

Fuente: Chasquibol (2017)

El presente trabajo de investigación fue uno de los objetivos del proyecto n. ${ }^{\circ}$ 020-2015-INIA-PNIA/UPMSI/IE “Microencapsulación de aceites de sacha inchi (Plukenetia volubilis y Plukenetia huayllabambana) y antioxidantes de la biodiversidad peruana. Contribución al desarrollo de alimentos funcionales conteniendo $\omega$-3 para prevenir enfermedades no transmisibles", del Programa Nacional de Innovación Agraria (PNIA) del Ministerio de Agricultura y Riego, con el financiamiento del Banco Mundial y del Instituto de Investigación Científica (IDIC) de la Universidad de Lima. 


\section{MATERIAL Y MÉTODOS}

\subsection{Muestra}

Las semillas de sacha inchi del ecotipo Plukenetia volubilis fueron recolectadas en la ciudad de Tarapoto (región San Martín) y las semillas del ecotipo Plukenetia huayllabambana en la provincia de Rodríguez de Mendoza (región Amazonas). La extracción de los aceites de sacha inchi se realizó, por prensado hidráulico en frío, en el Laboratorio de Máquinas e Instrumentos de la facultad de Ingeniería y Arquitectura de la Universidad de Lima. Los aceites se centrifugaron durante treinta minutos en una centrífuga trifásica de 220 V y de 2,9 HP a 3600 rpm en el Laboratorio de Grasas, Aceites y Alimentos Funcionales del Centro de Estudios e Innovación de Alimentos Funcionales (CEIAF). Finalmente, los aceites se almacenaron en frascos de color pardo y a la temperatura de $4^{\circ} \mathrm{C}$ para su posterior análisis y microencapsulación.

Las microcápsulas de aceite de sacha inchi (Plukenetia huayllabambana y Plukenetia volubilis) fueron obtenidas mediante secado por aspersión, empleando el equipo Mini Spray Dryer de marca Büchi modelo B-290. El proceso de microencapsulación y del presente trabajo fue realizado por el equipo de investigación del proyecto n. ${ }^{\circ}$ 020-2015-INIA-PNIA/ UPMSI/IE en el Laboratorio de Grasas, Aceites y Alimentos Funcionales del CEIAF de la facultad de Ingeniería y Arquitectura de la Universidad de Lima. Se emplearon dos muestras de aceite de sacha inchi y diez muestras de aceite de sacha inchi microencapsulado para cada uno de los ecotipos. Todos los análisis se realizaron por triplicado.

\subsection{Caracterización fisicoquímica}

\section{Humedad}

Se pesaron $3,00 \mathrm{~g} \pm 0,01 \mathrm{~g}$ de microcápsulas de los aceites de sacha inchi en la balanza de humedad Sartorius, modelo MA-30 a $100{ }^{\circ} \mathrm{C}$ y $1 \mathrm{~atm}$ de presión; las mediciones se realizaron por triplicado.

\section{Rendimiento}

El rendimiento de las microcápsulas se obtuvo según la fórmula siguiente:

Rendimiento(\%)= $\frac{\text { Microcápsulas obtenidas en el proceso de microencapsulación }(g)}{\text { Peso del material ingresante }(g)} \times 100 \%$

Índice de peróxido (I.P.)

Se disolvieron 2,00 \pm 0,01 g de muestra en una mezcla de ácido acético y cloroformo, se añadió solución de $\mathrm{KI}$ saturado, manteniendo las muestras en la oscuridad para que todo 
el yoduro se transforme en yodo con el oxígeno procedente de los peróxidos. El yodo formado se valoró con $\mathrm{Na}_{2} \mathrm{~S}_{2} \mathrm{O}_{3} 0.01 \mathrm{~N}$. Los resultados se expresaron en meq $\mathrm{O}_{2} / \mathrm{kg}$ aceite (Chasquibol, 2017) y se calcularon empleando la siguiente fórmula:

$$
\text { I.P }\left(\text { meq } \mathrm{O}_{2} / \mathrm{kg} \text { aceite }\right)=\frac{V(m L) \times N\left(\mathrm{Na}_{2} \mathrm{~S}_{2} \mathrm{O}_{3}\right) \times 1000}{\text { peso muestra }(g)}
$$

Donde:

$V(\mathrm{~mL})$ : Volumen gastado en la titulación $(\mathrm{mL})$

$\mathrm{N}$ : Normalidad corregida del $\mathrm{Na}_{2} \mathrm{~S}_{2} \mathrm{O}_{3}$

Las muestras fueron almacenadas en bolsas de aluminio a $-5{ }^{\circ} \mathrm{C}$, durante 120 días, tiempo en el cual se evaluó el índice de peróxido.

\section{Estabilidad oxidativa}

La estabilidad oxidativa del aceite de sacha inchi y de sus microencapsulados se realizó en el equipo Professional Metrohm Rancimat (modelo 892), empleándose entre $2,0 \mathrm{~g} \mathrm{a}$ $3,0 \mathrm{~g}$ de muestra y a las temperaturas de $70,80,90$ y $100^{\circ} \mathrm{C}$, con un caudal de aire de $20 \mathrm{~L} / \mathrm{h}$. La exposición de las muestras a estas temperaturas y flujo de aire a lo largo del tiempo descomponen a los aceites, desprendiéndose compuestos volátiles que se concentran en los vasos del equipo Rancimat (Chasquibol, 2017). Durante el periodo de inducción (índice de estabilidad oxidativa), ocurre la formación de hidroperóxidos en los aceites, generando la pérdida de calidad y de su vida útil. Los análisis se realizaron por triplicado, los resultados fueron recolectados y procesados mediante el software StabNet del equipo Professional Metrohm Rancimat (modelo 892) y mediante la técnica de extrapolación lineal en función de los índices de estabilidad oxidativa o periodos de inducción (PI) según las temperaturas de trabajo. Los valores de tiempo de vida útil de las muestras a $25^{\circ} \mathrm{C}$ se determinaron empleando la siguiente fórmula:

Tiempo de vida $=\mathrm{A} \times \exp (\mathrm{B} \times \mathrm{T})$

Donde:

A y B: Coeficientes de regresión en base a los PI

T: Temperatura del tiempo de vida establecido $\left(25^{\circ} \mathrm{C}\right)$

Energía de activación (Ea)

Se determinó a partir de la pendiente de la recta que resulta de representar el logaritmo natural de los valores del índice de estabilidad oxidativa (OSI) versus el inverso de la temperatura absoluta $(343 \mathrm{~K}, 353 \mathrm{~K}, 363 \mathrm{~K}, 373 \mathrm{~K})$. La dependencia de los valores de OSI con respecto a la temperatura termodinámica fue utilizada para determinar la energía de 
activación de las reacciones de oxidación de los aceites de sacha inchi microencapsulado y sin microencapsular. La energía de activación se determinó empleando la siguiente fórmula:

$$
\operatorname{Ln}(\mathrm{OSI})=\operatorname{Ln}\left(\frac{-\operatorname{Ln}(1-\alpha)}{Z}\right)+\frac{E a}{R T}
$$

Donde:

OSI: Índice de estabilidad oxidativa

R: Constante universal de los gases

Z: Factor prexponencial de la ecuación de Arrhenius

$\mathrm{T}$ : Temperatura (unidades en $\mathrm{K}$ )

\section{RESULTADOS Y DISCUSIÓN}

\subsection{Diseño experimental}

En la tabla 1 se muestra el diseño experimental empleado en el presente trabajo.

Tabla 1

Diseño experimental

\begin{tabular}{|c|c|c|}
\hline $\begin{array}{c}\mathrm{n} .^{\circ} \mathrm{de} \\
\text { muestra }\end{array}$ & Abreviación & Descripción \\
\hline 1 & ASIH & Aceite de sacha inchi Plukenetia huayllabambana sin microencapsular \\
\hline 2 & $\mathrm{ASIH}+\mathrm{GA}$ & $\begin{array}{l}\text { Aceite de sacha inchi Plukenetia huayllabambana microencapsulado } \\
\text { con goma arábiga (GA) }\end{array}$ \\
\hline 3 & $A S I H+G A+M D$ & $\begin{array}{l}\text { Aceite de sacha inchi Plukenetia huayllabambana microencapsula- } \\
\text { dos con goma arábiga (GA) y maltodextrina (MD) }\end{array}$ \\
\hline 4 & $A S I H+G A+M D+W P I$ & $\begin{array}{l}\text { Aceite de sacha inchi Plukenetia huayllabambana microencapsula- } \\
\text { dos con goma arábiga (GA), maltodextrina (MD) y proteína concen- } \\
\text { trada de suero de leche (WPI) }\end{array}$ \\
\hline 5 & ASIH + Hi-cap & $\begin{array}{l}\text { Aceite de sacha inchi Plukenetia huayllabambana microencapsulado } \\
\text { con almidón modificado Hi-cap }\end{array}$ \\
\hline 6 & ASIH + Capsul & $\begin{array}{l}\text { Aceite de sacha inchi Plukenetia huayllabambana microencapsulado } \\
\text { con almidón modificado Capsul }\end{array}$ \\
\hline 7 & ASIV & Aceite de sacha inchi Plukenetia volubilis sin microencapsular \\
\hline 8 & $A S I V+G A$ & $\begin{array}{l}\text { Aceite de sacha inchi Plukenetia volubilis microencapsulado con } \\
\text { goma arábiga (GA) }\end{array}$ \\
\hline 9 & $A S I V+G A+M D$ & $\begin{array}{l}\text { Aceite de sacha inchi Plukenetia volubilis microencapsulados con } \\
\text { goma arábiga (GA) y maltodextrina (MD) }\end{array}$ \\
\hline
\end{tabular}

(continúa) 
(continuación)

$\begin{array}{cll}10 & \text { ASIV + GA + MD + WPI } & \text { Aceite de sacha inchi Plukenetia volubilis microencapsulados con } \\ & \\ & & \\ & \text { goma arábiga (GA), maltodextrina (MD) y proteína concentrada de } \\ & \text { Aceite de sacha inchi Plukenetia volubilis microencapsulado con al- } \\ & \text { midón modificado Hi-cap } \\ & \text { Aceite de sacha inchi Plukenetia volubilis microencapsulado con al- } \\ & \text { midón modificado Capsul }\end{array}$

Fuente: Proyecto n. ${ }^{\circ}$ 020-2015-INIA-PNIA/UPMSI/IE del Programa Nacional de Innovación Agraria (PNIA) del Ministerio de Agricultura y Riego, Banco Mundial y el Instituto de Investigación Científica (IDIC) de la Universidad de Lima.

\subsection{Porcentaje de humedad y rendimiento}

En la figura 2, se observa que el porcentaje de humedad de las microcápsulas de los aceites de sacha inchi (Plukenetia huayllabambana y Plukenetia volubilis) son menores del 10 \% establecido para productos deshidratados (Huntington, 2007; Pastuña y otros, 2016). Las microcápsulas con Hi-cap como material de pared son las que presentan menor porcentaje de humedad para ambos aceites a un nivel de confianza del $95 \%$, comparado con la goma arábica que contiene mayor porcentaje de humedad a un nivel de confianza del $95 \%$.

En la figura 3, se observa que los porcentajes de rendimiento son menores del $40 \%$, que es el rendimiento esperado en procesos de microencapsulación en laboratorio. La microcápsula de aceite de sacha inchi Plukenetia huayllabambana con material de pared ternaria (GA + MD + WPI) fue la que presentó mayor rendimiento $(39,88 \% \pm 1,2 \%)$, con un nivel de confianza del $95 \%$.

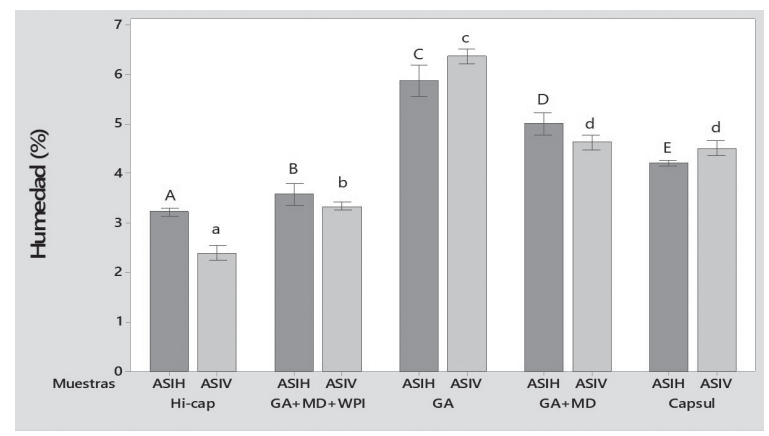

Figura 2. Porcentaje humedad de las microcápsulas de los aceites de sacha inchi

Fuente: Proyecto n. ${ }^{\circ}$ 020-2015-INIA-PNIA/UPMSI/IE del Programa Nacional de Innovación Agraria (PNIA) del Ministerio de Agricultura y Riego, Banco Mundial y el Instituto de Investigación Científica (IDIC) de la Universidad de Lima. 


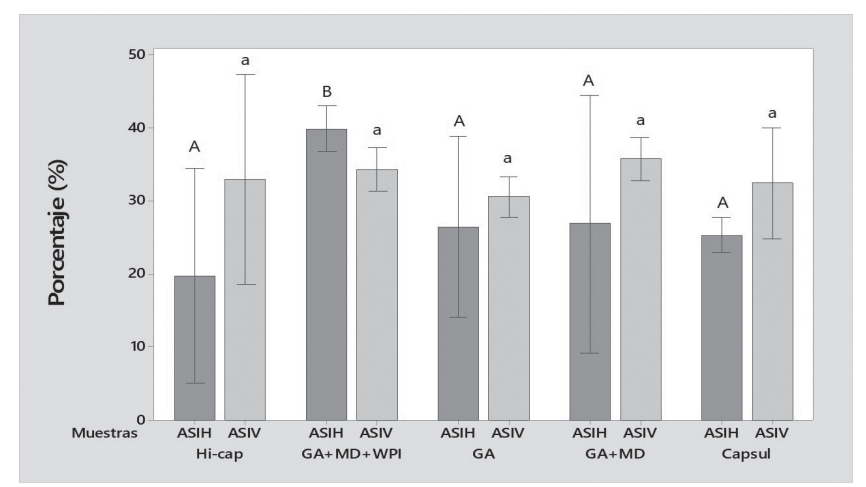

Figura 3. Porcentaje de rendimiento de las microcápsulas de los aceites de sacha inchi

Fuente: Proyecto n. ${ }^{\circ}$ 020-2015-INIA-PNIA/UPMSI/IE del Programa Nacional de Innovación Agraria (PNIA) del Ministerio de Agricultura y Riego, Banco Mundial y el Instituto de Investigación Científica (IDIC) de la Universidad de Lima.

\section{3 Índice de peróxidos}

En la tabla 2 se muestran los índices de peróxidos de las microcápsulas durante el tiempo de almacenamiento. Se observa que, a los setenta días, el índice de peróxido se encuentran dentro de los valores establecidos por la Norma Técnica Peruana de Sacha Inchi (Norma Técnica Peruana NTP 151.400, 2014), que establece un valor máximo de 10 meq $\mathrm{O}_{2} / \mathrm{kg}$ aceite; mientras que, a los 120 días de almacenamiento, las muestras microencapsuladas con Hi-cap y con la mezcla ternaria (GA + MD + WPI) son las únicas que se encuentran dentro de los límites establecidos por la Norma Técnica Peruana de Sacha Inchi.

\section{Tabla 2}

Índice de peróxidos de las microcápsulas de aceite de sacha inchi

\begin{tabular}{llccc}
\hline Aceites & $\begin{array}{l}\text { Material } \\
\text { de pared }\end{array}$ & 0 & 70 & 120 \\
\hline Aceite de sacha inchi & Hi-cap & $0,0000 \pm 0,0000$ & $0,0000 \pm 0,0000$ & $4,9511 \pm 0,6601$ \\
Plukenetia & GA+MD+WPI & $2,9763 \pm 0,0102$ & $5,5349 \pm 0,0019$ & $9,1192 \pm 0,0032$ \\
huayllabambana & GA & $2,9856 \pm 0,0079$ & $8,3026 \pm 0,0017$ & $11,0483 \pm 0,6575$ \\
& GA+MD & $3,9148 \pm 0,0957$ & $7,3860 \pm 0,0019$ & $10,2625 \pm 0,0010$ \\
& Capsul & $6,4563 \pm 0,0030$ & $7,8530 \pm 0,1269$ & $12,1627 \pm 0,6610$ \\
\hline & & & & (continúa)
\end{tabular}


(continuación)

\begin{tabular}{lllll}
\hline Aceite de sacha inchi & Hi-cap & $0,0000 \pm 0,0000$ & $0,0000 \pm 0,0000$ & $4,9546 \pm 0,6601$ \\
$\begin{array}{llll}\text { Plukenetia volubilis } \\
\text { GA+MD+WPI }\end{array}$ & $2,9763 \pm 0,0102$ & $6,2974 \pm 0,5514$ & $9,1219 \pm 0,0014$ \\
& GA & $2,9856 \pm 0,0079$ & $6,4546 \pm 0,0038$ & $9,1298 \pm 0,0070$ \\
& GA+MD & $3,9148 \pm 0,0957$ & $5,6377 \pm 0,1727$ & $7,9793 \pm 0,0012$ \\
& Capsul & $6,4563 \pm 0,0030$ & $9,8995 \pm 0,0099$ & $12,1667 \pm 0,6508$ \\
\hline
\end{tabular}

Fuente: Proyecto n. 020-2015-INIA-PNIA/UPMSI/IE del Programa Nacional de Innovación Agraria (PNIA) del Ministerio de Agricultura y Riego, Banco Mundial y el Instituto de Investigación Científica (IDIC) de la Universidad de Lima.

\subsection{Estabilidad oxidativa}

Los altos grados de insaturaciones presentes en el aceite de sacha inchi están asociados con un menor índice de estabilidad oxidativa. La estabilidad oxidativa se define como la resistencia a la oxidación bajo condiciones establecidas y se expresa como el periodo que corresponde al tiempo desde que la oxidación procede lentamente hasta un inesperado incremento en la reacción oxidativa que es llamado periodo de inducción (PI) (Velasco et al., 2009).

El PI indica la iniciación de la formación de hidroperóxidos que contienen dobles enlaces trans conjugados, y el tiempo de estabilidad de los aceites de sacha inchi indica la formación de compuestos orgánicos aromáticos de cadena corta (como aldehídos, cetonas, alcoholes, y ácidos grasos de cadena corta) que son perjudiciales para la salud (Sherwin, 1978). Los periodos de inducción y los tiempos de estabilidad tienden a duplicarse por cada $10{ }^{\circ} \mathrm{C}$ de aumento en la temperatura debido a la elevada insaturación de los aceites de sacha inchi, como ha sido comprobado en otros trabajos de investigación (Villanueva et al, 2013; Rodríguez et al, 2015). Los tiempos de inducción de los aceites de sacha inchi sin microencapsular y microencapsulado obtenidos en el equipo Rancimat a las temperaturas de trabajo $70^{\circ} \mathrm{C}, 80^{\circ} \mathrm{C}, 90^{\circ} \mathrm{C}$ y $100^{\circ} \mathrm{C}$ se muestran en la tabla 3.

Los resultados de la tabla 3 se emplearon para determinar el tiempo de vida útil de los aceites sin microencapsular y microencapsulados a la temperatura de $25^{\circ} \mathrm{C}$ (punto rojo en la recta verde), obtenido mediante el software StabNet del equipo Rancimat (figura 4). El tiempo de vida útil extrapolada a $25^{\circ} \mathrm{C}$ (tabla 4), al igual que los PI, se encuentran en una línea de tendencia exponencial debido a que la velocidad de oxidación es exponencialmente proporcional a la temperatura y la vida útil de los aceites disminuye logarítmicamente con el aumento de la temperatura (Frankel, 1998). Además, el elevado porcentaje de ácidos grasos poliinsaturados en los aceites de sacha inchi influyen en su estabilidad oxidativa. por consiguiente, el tiempo de vida útil extrapolada a $25^{\circ} \mathrm{C}$ (tabla 4) fue mucho mayor a 1000 horas para los microencapsulados con Hi-cap para ambos aceites, mientras que los tiempos de vida más bajos fueron con el material de pared Capsul. 
Tabla 3

Tiempo de inducción de los aceites de sacha inchi sin microencapsular y microencapsulados

\begin{tabular}{|c|c|c|c|c|c|}
\hline \multirow[t]{2}{*}{ Aceites } & \multirow[t]{2}{*}{ Muestras } & \multicolumn{4}{|c|}{ Tiempo de inducción (h) } \\
\hline & & $70^{\circ} \mathrm{C}$ & $80^{\circ} \mathrm{C}$ & $90^{\circ} \mathrm{C}$ & $100{ }^{\circ} \mathrm{C}$ \\
\hline \multirow{6}{*}{$\begin{array}{l}\text { Aceite de sacha } \\
\text { inchi Plukenetia } \\
\text { huayllabambana }\end{array}$} & Hi-cap & $82,75 \pm 0,74$ & $34,08 \pm 0,70$ & $17,11 \pm 0,40$ & $8,66 \pm 0,14$ \\
\hline & $G A+M D+W P I$ & $62,14 \pm 3,23$ & $27,72 \pm 0,96$ & $14,22 \pm 0,70$ & $6,81 \pm 0,12$ \\
\hline & GA & $31,10 \pm 1,09$ & $13,86 \pm 1,20$ & $4,94 \pm 0,47$ & $2,48 \pm 0,29$ \\
\hline & $\mathrm{GA}+\mathrm{MD}$ & $33,26 \pm 4,85$ & $14,13 \pm 0,16$ & $5,64 \pm 0,15$ & $2,90 \pm 0,06$ \\
\hline & ASIH & $71,31 \pm 3,01$ & $32,46 \pm 0,90$ & $18,40 \pm 0,85$ & $9,28 \pm 0,78$ \\
\hline & Capsul & $43,76 \pm 1,78$ & $21,21 \pm 0,08$ & $12,60 \pm 2,09$ & $5,81 \pm 0,16$ \\
\hline \multirow{6}{*}{$\begin{array}{l}\text { Aceite de sacha } \\
\text { inchi Plukenetia } \\
\text { volubilis }\end{array}$} & Hi-cap & $95,32 \pm 2,48$ & $40,31 \pm 1,27$ & $18,36 \pm 0,36$ & $8,89 \pm 0,29$ \\
\hline & $G A+M D+W P I$ & $84,62 \pm 2,95$ & $37,65 \pm 0,90$ & $18,66 \pm 0,25$ & $9,70 \pm 0,15$ \\
\hline & GA & $78,00 \pm 1,57$ & $38,61 \pm 1,49$ & $18,70 \pm 0,61$ & $9,26 \pm 0,17$ \\
\hline & $\mathrm{GA}+\mathrm{MD}$ & $74,53 \pm 0,99$ & $32,06 \pm 0,40$ & $16,41 \pm 0,33$ & $8,18 \pm 0,11$ \\
\hline & ASIV & $41,76 \pm 6,31$ & $19,38 \pm 2,93$ & $9,23 \pm 0,28$ & $4,60 \pm 0,35$ \\
\hline & Capsul & $34,45 \pm 11,03$ & $16,23 \pm 0,30$ & $10,08 \pm 1,79$ & $6,59 \pm 0,01$ \\
\hline
\end{tabular}

Fuente: Proyecto n. ${ }^{\circ}$ 020-2015-INIA-PNIA/UPMSI/IE del Programa Nacional de Innovación Agraria (PNIA) del Ministerio de Agricultura y Riego, Banco Mundial y el Instituto de Investigación Científica (IDIC) de la Universidad de Lima.

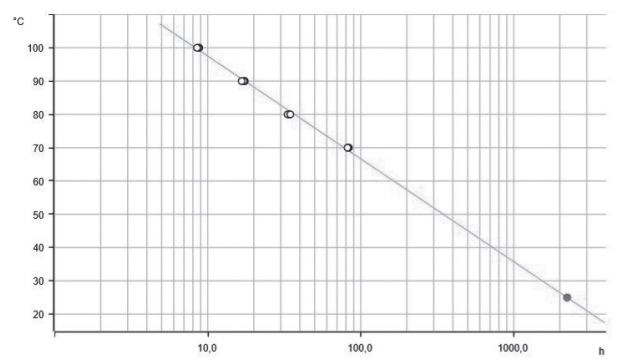

Figura 4. Extrapolación del PI y tiempo de vida útil del aceite de sacha inchi Plukenetia huayllabambana microencapsulado con Hi-cap como material de pared

Fuente: Proyecto n. ${ }^{\circ}$ 020-2015-INIA-PNIA/UPMSI/IE del Programa Nacional de Innovación Agraria (PNIA) del Ministerio de Agricultura y Riego, Banco Mundial y el Instituto de I nvestigación Científica (IDIC) de la Universidad de Lima. 
Tabla 4

Tiempo de vida útil extrapolada a $25^{\circ} \mathrm{C}$ de los aceites de sacha inchi sin microencapsular y microencapsulado

\begin{tabular}{lll}
\hline Aceites & Material de pared & Tiempo de vida útil extrapolada a $25^{\circ} \mathrm{C}$ \\
\hline Aceite de sacha inchi & Hi-cap & $2232 \mathrm{~h}=0,25$ años \\
Plukenetia huayllabambana & GA+MD+WPI & $1614 \mathrm{~h}=0,18$ años \\
& GA & $1507 \mathrm{~h}=0,17$ años \\
& GA+MD & $1457 \mathrm{~h}=0,16$ años \\
& ASIH & $1388 \mathrm{~h}=0,15$ años \\
Caceite de sacha inchi & Hi-cap & $838,3 \mathrm{~h}=0,09$ años \\
Plukenetia volubilis & GA+MD+WPI & $3464 \mathrm{~h}=0,39$ años \\
& GA & $2019 \mathrm{~h}=0,23$ años \\
GA+MD & $1918 \mathrm{~h}=0,21$ años \\
& ASIV & $1895 \mathrm{~h}=0,21$ años \\
Capsul & $1105 \mathrm{~h}=0,12$ años \\
\hline
\end{tabular}

Fuente: Proyecto n. ${ }^{\circ}$ 020-2015-INIA-PNIA/UPMSI/IE del Programa Nacional de Innovación Agraria (PNIA) del Ministerio de Agricultura y Riego, Banco Mundial y el Instituto de Investigación Científica (IDIC) de la Universidad de Lima.

\subsection{Energía de activación (Ea)}

Según Blaine y Savage (1992) y García-Ochoa y otros (1989), la adición de los radicales de oxígeno a los ácidos grasos se produce en los dobles enlaces entre los átomos de carbono, siguiendo cinéticas de primer orden. Además, el efecto de la temperatura en los valores del índice de estabilidad oxidativa obtenidos por el método de Rancimat puede ser estudiado aplicando tanto la ecuación empírica como el modelo cinético (Navas, 2010). Se puede obtener la energía de activación de la reacción con el fin de poder comprender mejor el proceso de oxidación de los aceites vírgenes como es el caso de los aceites de sacha inchi. Para hallar la energía de activación, se empleó el diagrama de la inversa de la temperatura absoluta versus el logaritmo natural de los índices de estabilidad oxidativa de los aceites de sacha inchi sin microencapsular y microencapsulados (figura 5). 


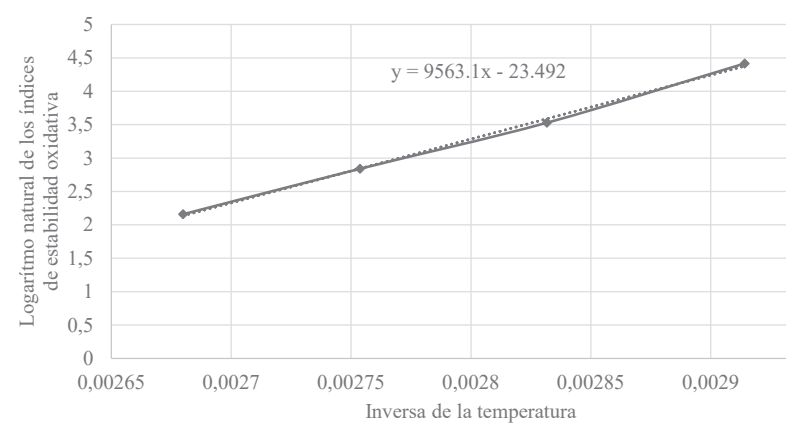

Figura 5. Gráfico de la inversa de la temperatura absoluta y el logaritmo natural de los índices de estabilidad oxidativa de los aceites de sacha inchi sin microencapsular y microencapsulados.

Fuente: Proyecto n. ${ }^{\circ}$ 020-2015-INIA-PNIA/UPMSI/IE del Programa Nacional de Innovación Agraria (PNIA) del Ministerio de Agricultura y Riego, Banco Mundial y el Instituto de Investigación Científica (IDIC) de la Universidad de Lima.

Los resultados de las energías de activación se indican en la tabla 5. Se observa que la energía de activación es mayor en las microcápsulas para ambos aceites, con excepción del material de pared Capsul. La energía de activación para el aceite de sacha inchi ( $P$. huayllabambana) fue de $71,20 \mathrm{KJ} / \mathrm{mol}$, mientras que para el aceite de sacha inchi microencapsulado con goma arábica fue de $91,78 \mathrm{KJ} / \mathrm{mol}$. Para el aceite de sacha inchi ( $P$. volubilis) su energía de activación fue de $78,35 \mathrm{KJ} / \mathrm{mol}$, mientras que para el aceite de sacha inchi microencapsulado con Hi-cap fue de $84,18 \mathrm{KJ} / \mathrm{mol}$.

\section{Tabla 5}

Energía de activación de los aceites de sacha inchi sin microencapsular y microencapsulados

\begin{tabular}{lcc}
\hline Material de pared & EA (kJ/mol) \\
& $\begin{array}{c}\text { Aceite de sacha inchi } \\
\text { Plukenetia huallabambana }\end{array}$ & $\begin{array}{c}\text { Aceite de sacha inchi } \\
\text { Plukenetia volubilis }\end{array}$ \\
\hline Hi-cap & 79,51 & 84,18 \\
GA+MD+WPI & 77,73 & 76,71 \\
GA & 91,78 & 75,74 \\
GA+MD & 87,76 & 77,75 \\
Sin microencapsular & 71,20 & 78,35 \\
Capsul & 69,97 & 58,06 \\
\hline
\end{tabular}

Fuente: Proyecto n. ${ }^{\circ}$ 020-2015-INIA-PNIA/UPMSI/IE del Programa Nacional de Innovación Agraria (PNIA) del Ministerio de Agricultura y Riego, Banco Mundial y el Instituto de Investigación Científica (IDIC) de la Universidad de Lima. 


\section{CONCLUSIONES}

El porcentaje de humedad de las microcápsulas de aceite de sacha inchi (Plukenetia huayllabambana y Plukenetia volubilis) cumple con los requisitos de productos deshidratados y porcentaje de rendimiento obtenido por el método de spray dryer.

Las microcápsulas formuladas con material de pared Hi-cap se mantienen estables durante el tiempo de almacenamiento, porque el valor del índice de peróxidos se encuentra dentro del límites establecidos por la normativa peruana.

El proceso de microencapsulación protege a los aceites del proceso de oxidación, aumentando su tiempo de vida útil a la temperatura de $25^{\circ} \mathrm{C}$. El aceite de sacha inchi Plukenetia huayllabambana de 1388 h aumentó su tiempo de vida útil a $2232 \mathrm{~h}$ en los aceites microencapsulados con material de pared Hi-cap, mientras que el aceite de sacha inchi Plukenetia volubilis de $1105 \mathrm{~h}$ aumentó su tiempo de vida útil a $25^{\circ} \mathrm{C}$ a $3464 \mathrm{~h}$ en los aceites microencapsulados con material de pared Hi-cap.

La energía de activación para el aceite de sacha inchi (P. huayllabambana) fue de 71,20 $\mathrm{KJ} / \mathrm{mol}$, aumentando a $91,78 \mathrm{KJ} / \mathrm{mol}$ para el aceite microencapsulado con goma arábica, mientras que para el aceite de sacha inchi ( $P$. volubilis) su energía de activación fue de $78,35 \mathrm{KJ} / \mathrm{mol}$, aumentando a $84,18 \mathrm{KJ} / \mathrm{mol}$ para el aceite microencapsulado con Hi-cap.

La determinación de la estabilidad oxidativa para los aceites de sacha inchi y para otros aceites vegetales debe establecerse como un parámetro de calidad importante para asegurar el tiempo de vida bajo determinadas condiciones de almacenamiento (temperatura, humedad, entre otros), manteniendo sus características nutricionales, funcionales y sensoriales.

\section{REFERENCIAS}

Ancira, R. (2008). Omega 3: los secretos curativos del eslabón perdido de la salud. México, D. F.: Editorial Kindle.

Blaine, S. y Savage, P. (1992). Reaction Pathways in Lubricant Degradation, 3, Reaction Model for $n$-Hexadecane autoxidation. Industrial \& engineering chemistry research, 31(1), 69-75.

Bondioli, P. y Della Bella, L. (2006). Alpha linolenic acid rich oils. Composition of Plukenetia volubilis (sacha inchi) oil from Peru. Rivista italiana delle Sostanze Grasse, 83(3), 120.

Cabré, P. y Massó, A. (2014). Determinación de la estabilidad a la oxidación de aceites y grasas por el método Rancimat. Barcelona: Massó Analítica.

Chasquibol, N. (2017). Caracterización, análisis sensorial y estabilidad oxidativa del aceite de sacha inchi (Plukenetia huayllabambana). Autenticidad de aceites comerciales de sacha inchi. Lima: UNMSM. 
Chasquibol, N.; Chellah, D.; Yacono, J.; Guinda, Á.; Moreda, W.; Gómez-Roca, R. y Pérez Camino, M. (2014). Characterization of Glyceridic and Unsaponifi able Compounds of sacha inchi (Plukenetia huayllabambana L.) Oils. Journal of agricultural and food chemistry, 62(41), 10162-10169. doi:10.1021/jf5028697

Chasquibol, N.; Moreda, W.; Yácono, J. C. y Pérez-Camino, M. C. (2012). Preliminary characterization studies on sacha inchi (Plukenetia volubilis L.) seeds and oils grown in San Martín, Peru. $20^{\text {th }}$ International Symposium on Plant Lipids ISPL.

Chasquibol, N.; Yacono, J.; Pérez Camino, M.; Del Águila, C. y Moreda, W. (2014). Specific markers of the addition of other seed oils to commercial extra virgin sacha inchi oils. $12^{\text {th }}$ Euro Fed Lipids Congress. European Federation for the Science and Technology of Lipids. Montpellier, Francia.

Frankel, E. N. (1998). Lipid oxidation. Dundee, Scotland: Oily Press.

García-Ochoa, F.; Romer, A. y Querol, A. (1989). Modeling of the Thermal n-Octane Oxidation in the Liquid Phase. Industrial and Engineering Chemical Research, 28(1), 43-48.

Garmendia, F.; Pando, R. y Ronceros, G. (2011). Efecto del aceite de sacha inchi (Plukenetia volúbilis l) sobre el perfil lipídico en pacientes con hiperlipoproteinemia. Revista Peruana de Medicina Experimental y Salud Pública, 28(4), 628-632.

Gonzales, G. F. y Gonzales, C. (2014). A randomized, double-blind placebo-controlled study on acceptability, safety and efficacy of oral administration of sacha inchi oil (Plukenetia volubilis L.) in adult human subjects. Food and Chemical Toxicology, 65, 168-176.

Gorriti, A.; Arroyo, J.; Quispe, F.; Cisneros, B.; Condorhuam M.; Almora Y. y Chumpitaz, V. (2010). Toxicidad oral a 60 días del aceite de sacha inchi (Plukenetia volubilis L.) y linaza (Linum usitatissimum L.) y determinación de la dosis letal 50 en roedores. Revista Peruana de Medicina Experimental y Salud Pública, 27(3), 352-60.

Guillén, M. D.; Ruiz, A.; Cabo, N.; Chirinos, R. y Pascual, G. (2003). Characterization of sacha inchi (Plukenetia volubilis L.) Oil by FTIR Spectroscopy and 1H NMR. Comparison with Linseed Oil. Journal of the American Oil Chemists' Society, 80(8), 755-762.

Huamán J.; Chávez K. y Castañeda E. (2008). Efecto de la Plukenetia volubilis Linneo (sacha inchi) en la trigliceridemia posprandial. An. Fac. med, 69(4), 263-266.

Huntington, D. (2007). The Influence of the Spray Drying Process on Product. Drying technology, 22(6), 1261-1287.

Liua, Q.; Xua, Y.; Zhanga, P.; Na, Z.; Tanga, T. y Shia, Y. (2014). Chemical composition and oxidative evolution of sacha inchi (Pluketia volubilis L.) oil from Xishuangbanna (China). Grasas Aceites, 65(1). doi: http://dx.doi.org/10.3989/gya.075713. 
Muñoz Jáuregui, A.; Alvarado-Ortiz, C.; Castañeda, B.; Lizaraso, F.; Barnett, E.; Cárdenas, L. y Manco, E. (2013). Estudio Nutricional de Plukentia Huayllabambana sp.nov. Revista Sociedad Química del Perú, 79(1), 47-56.

Navas, P. (2010). Componentes minoritarios y propiedades antioxidantes de aceites vírgenes y tortas residuales obtenidos por presión en frío a partir de fuentes vegetales convencionales y no convencionales. [Tesis de posgrado, Universidad de Castilla La Mancha, España]. Repositorio institucional de la Universidad de Castilla La Mancha: http://hdl.handle.net/10578/2824

Norma Técnica Peruana NTP 151.400 (2014). Aceite de Sacha Inchi.

Pastuña Pullutasig, A.; López Hernández, O.; Debut, A.; Vaca, A.; Rodríguez Leyes, E.; Vicente, R.; ... y Tapia Hernández, F. (2016). Microencapsulación de aceite de sacha inchi. Revista Colombiana de Ciencias Químico-Farmacéuticas, 45(3), 422-437.

Páucar Menacho, L.; Salvador Reyes, R.; Guillén Sánchez, J.; Capa Robles, J. y Moreno Rojo, C. (2015). Estudio comparativo de las características físico-químicas del aceite de sacha inchi (Plukenetia volubilis L.), aceite de oliva (Olea europaea) y aceite crudo de pescado. Scientia Agropecuaria, 6(4), 279-290.

Rodríguez, G.; Villanueva, E.; Glorio, P. y Baquerizo, M. (2015). Estabilidad oxidativa y estimación de la vida útil del aceite de sacha inchi (Plukenetia volubilis L.). Scientia agropecuaria, 6(3), 155-163.

Sherwin, E. (1978). Oxidation and antioxidants in fat and oil processing. Journal of the American Oil Chemists' Society, 55(11), 809-814.

Velasco, J.; Dobarganes, C.; Holgado, F. y Marquez Ruiz, G. (2009). A follow-up oxidation study in dried microencapsulated oils under the accelerated conditions of the Rancimat test. Food Research International, 42(1), 56-62.

Villanueva López, E.; Castillo Benites, D. y Rodríguez Páucar, G. (2013). Influencia de los parámetros Rancimat sobre la determinación del índice de estabilidad oxidativa del aceite de Sesamum indicum L. Scientia Agropecuaria, 4(3), 173-180. 